\title{
Combining Scanning Electron Microscopy and Light Microscopy to Study How Blood Cell Directed-architecture Influences Nanoparticle Penetration into Clots
}

Cammy Truong, Courtney Cazzola, Sara Benzow, Austen Norberg, Ashlee Chramega, Heather Owen and Julie Oliver

University of Wisconsin-Milwaukee, Milwaukee, Wisconsin, United States

Activated platelets are a key component of the arterial thrombi responsible for heart attack and stroke. Tissue plasminogen activator (tPA) is currently the only FDA-approved drug for ischemic stroke. It works by dissolving fibrin, thus breaking down the clot and restoring blood flow to the brain. However, it must be administered within 4.5 hours of the onset of stroke. This leaves a significant number of patients who are ineligible for intervention and who have poor clinical outcomes. Additionally, tPA is not injury sitespecific; consequently, the side effects range from mild to severe ${ }^{1,2,3}$. We are investigating targeting activated platelets in occlusive thrombi as an alternative therapy for ischemic stroke.

The binding of plasma fibrinogen to its platelet surface receptor is absolutely dependent on cell activation and could therefore function in selective targeting of activated platelets. Additionally, when fibrinogen receptors are cross-linked by ligand, they engage the actin cytoskeleton and clear from the edges of platelets or groups of platelets, thereby exposing additional unoccupied receptors ${ }^{4,5,6}$. We propose that fibrinogen-conjugated nanoparticles can be used to target activated platelets rather than fibrin within an existing occlusive clot as a means to restore blood flow in the vessel. In our model, selection for activated platelets over quiescent circulating platelets is critical in order to minimize bleeding complications that are especially dangerous in ischemic brain.

Previous studies have demonstrated the specific targeting of fibrinogen-conjugated, gold-coated magnetite nanoparticles $\left(\mathrm{FGN}-\mathrm{cAu}-\mathrm{Fe}_{3} \mathrm{O}_{4}\right)$ to activated platelets in a purified platelet system. When followed by exposure to an oscillating magnetic field, the activated platelets in aggregates were disrupted ${ }^{7}$. Plateletrich plasma (PRP) and whole blood (WB) systems are more complex in terms of both the protein and cell components, but are also more representative of the physiological system. Developing $\mathrm{FGN}-\mathrm{cAu}-\mathrm{Fe}_{3} \mathrm{O}_{4}$ into an effective therapy will depend on how efficiently the nanoparticles penetrate into clots and their final concentration inside clots. We have used the robust model of fibrinogen-conjugated gold nanoparticles (FGN-cAu 18 ) to examine the effect that the 3-dimensional network of fibrin and blood cell components found in PRP and WB clots has on nanoparticle penetration. We hypothesized that different fibrin architecture resulting from clotting at different physiological thrombin concentrations determines the extent of nanoparticle penetration into clots. PRP and WB were clotted in vitro using low (1 nM) and high $(10 \mathrm{nM})$ doses of thrombin followed by soaking in FGN-cAu18. Slices and medial cross-sections were analyzed by scanning electron microscopy to observe both the topography and interior of the clots. Frozen cross-sections were treated with silver enhancement and analyzed by light microscopy to quantify the depth of nanoparticle penetration observed in different donors.

From our experiments, we have found: First, the arrangement of cells through the clots differed between the systems (fig.1). In PRP, fibrin and platelets were distributed throughout the clot. In WB clots, a meshwork of fibrin and platelet aggregates were observed on the clot surface, and the interior consisted of closely packed, compressed polyhedral erythrocytes, with very little fibrin present. Second, the dose of thrombin available during clotting significantly influences the density of clot structure. High thrombin doses produced a dense network of small diameter fibrin filaments, and low doses produced a more open 
network of large diameter fibers. Third, the extent of nanoparticle penetration was inversely related to the density of the fibrin network and the number of activated platelets and erythrocytes. Fourth, while variation between donors was observed its effect on nanoparticle penetration was largely overcome at high thrombin concentrations where there was a dense clot structure and an overall lower degree of nanoparticle penetration into clots (fig.2). These results provide important insight into the development of a nanoparticle-based therapeutic agent for treating ischemic stroke.

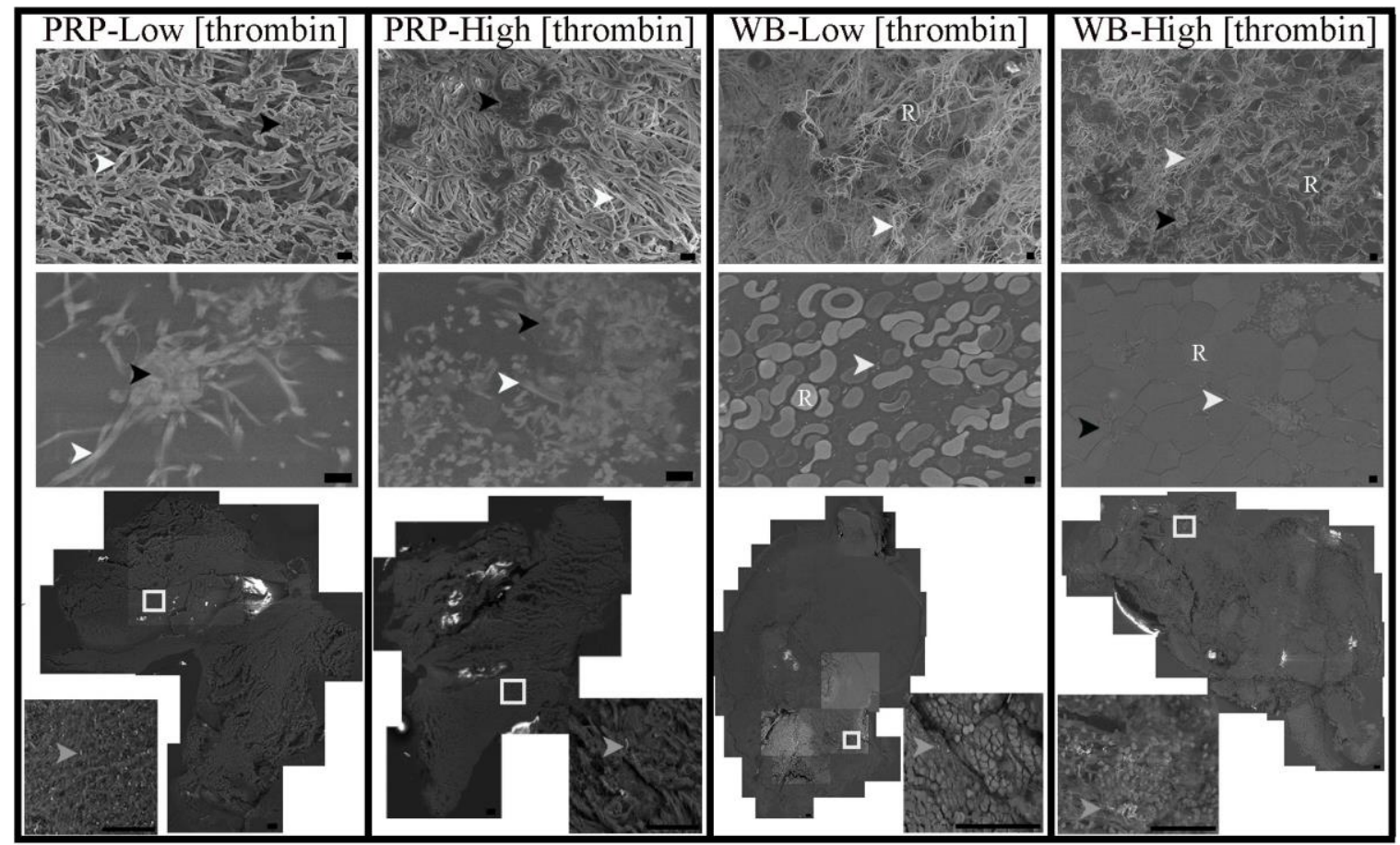

Figure 1. SEM images of FGN-cAu18 labeled PRP and WB clots formed at low and high thrombin concentrations. Surface view (top row), interior view (middle row) and montages (bottom row) with high magnification micrograph of nanoparticles at the deepest site found inside clots. White arrows, black arrows, and grey arrows indicate fibrin, activated platelets and FGN-cAu18, respectively and $\mathrm{R}$ indicates erythrocytes. Bar equals $1 \mu \mathrm{m}$ (top and middle row) and bar equals $50 \mu \mathrm{m}$ (bottom row). 


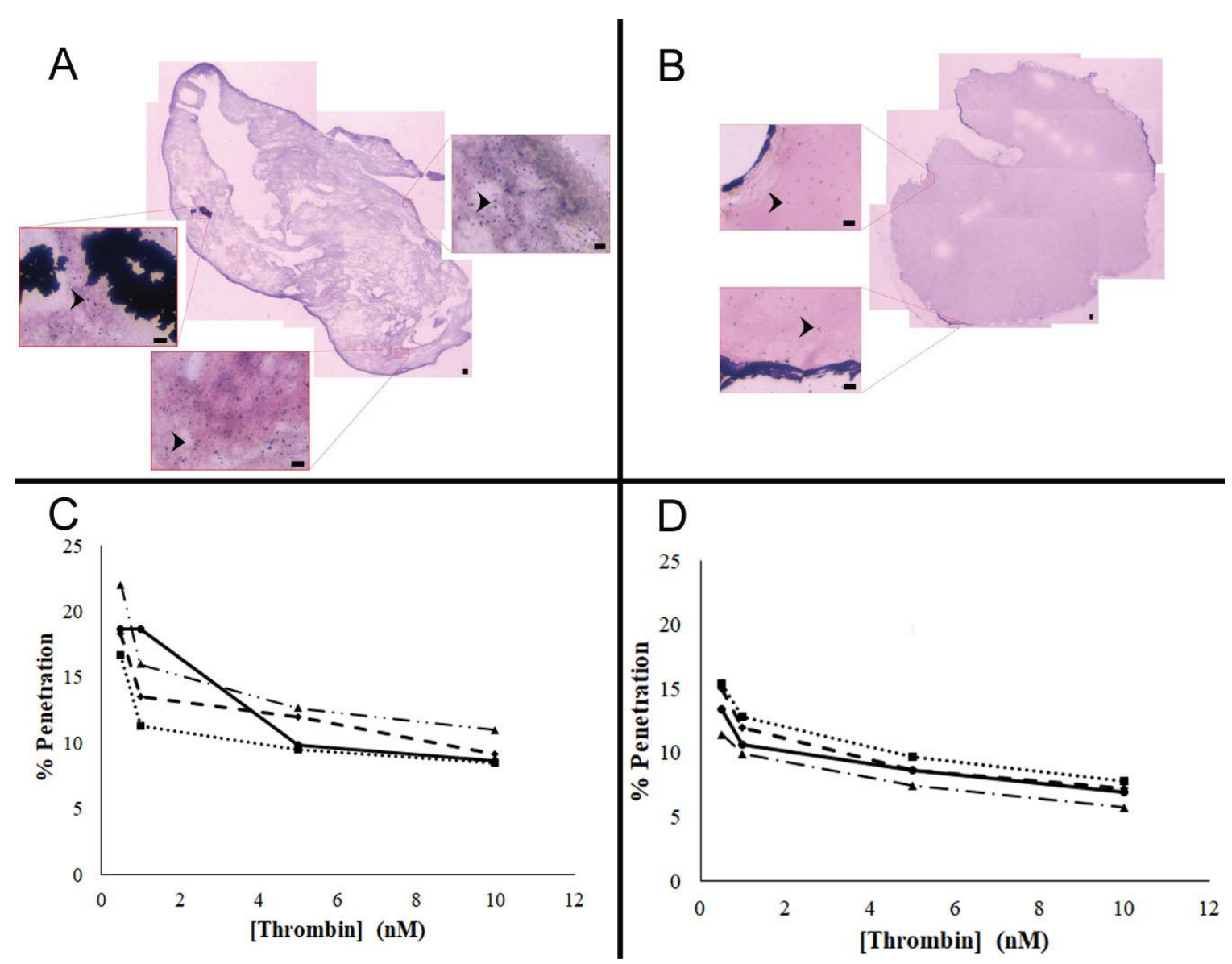

Figure 2. Reconstructed bright field images of fibrinogen conjugated-gold nanoparticle labeled PRP (A) and WB (B) clot sections and the quantification of nanoparticle penetration (percentage of total diameter) into PRP (C) and WB (D) clots from four different donors. Arrows indicates the presence of silver deposited on fibrinogen conjugated-gold nanoparticle nuclei. Scale bars equal $100 \mu \mathrm{m}$.

References

[1] Macfarlane, R, G., and Biggs, R. 1948. Fibrinolysis. Its Mechanism and Significance. Blood. 3 (10): 1167-1187

[2] Collen, D. 1996. Fibrin-Selective Thrombolytic Therapy for Acute Myocardial Infarction. Circulation. 93 (5): 857-865

[3] Demaerschalk, B, M., Demaerschalk, D, O., Adeoye, O, M., Demchuk, A, M., Fugate, J, E., Grotta, J, C., Khalessi, A, A., Levy, E, I., Palesch, Y, Y., Prabhakaran, S., Saposnik, G., Smith, E, E. 2016. Scientific Rationale for the Inclusion and Exclusion Criteria for Intravenous Alteplase in Acute Ischemic Stroke A Statement for Healthcare Professionals from the American Heart Association/American Stroke Association. Stroke. 47: 581-641

[4] Fox, J, E, B. 1985. The Organizations of Platelet Contractile Protein, Chapt. 13 in: Platelet Membrane Glycoproteins. J. N. Geogre, A. T. Nurden and D. R. Phillips (Eds.) Plenum Press, New York, pp. $273-$ 298.

[5] Kunicki, T, J. 1985. Organization of Glycoproteins with the Platelet Plasma Membrane, Chapt. 4 in: Platelet Membrane Glycoproteins. J. N. Geogre, A. T. Nurden and D. R. Phillips (Eds.) Plenum Press, New York, pp. 87-104. 
[6] Oliver, J, A., and Albrecht, R, M. 1987. Colloid Gold Labeling of Fibrinogen Receptor in Epinephrineand ADP-Activated Platelet Suspensions. Scanning Microsc. 1(2): 745-756.

[7] Krystofiak, E, S. 2013. Fibrinogen-Conjugated Gold-Coated Magnetite Nanoparticles for Antiplatelet Therapy. Doctoral Dissertation, University of Wisconsin- Milwaukee. 